OPEN ACCESS

Edited by:

Ingrid Melle,

University of Oslo, Norway

Reviewed by:

Rune A. Kroken,

Haukeland University

Hospital, Norway

Yikang Zhu,

Shanghai Jiao Tong University, China

*Correspondence:

Shuiyuan Xiao

xiaosy@csu.edu.cn

Junqun Fang

fangjun3497@163.com

Specialty section: This article was submitted to

Schizophrenia,

a section of the journal

Frontiers in Psychiatry

Received: 07 August 2021 Accepted: 28 January 2022 Published: 24 February 2022

Citation: Ouyang F, He J, Cheng X, Zhou W,

Xiao $S$ and Fang J (2022) Antipsychotic-Related Risks of Type 2 Diabetes Mellitus in Enrollees With Schizophrenia in the National Basic

Public Health Service Program in Hunan Province, China.

Front. Psychiatry 13:754775.

doi: 10.3389/fpsyt.2022.754775

\section{Antipsychotic-Related Risks of Type 2 Diabetes Mellitus in Enrollees With Schizophrenia in the National Basic Public Health Service Program in Hunan Province, China}

\author{
Feiyun Ouyang ${ }^{1}$, Jun $\mathrm{He}^{1}$, Xunjie Cheng ${ }^{2}$, Wei Zhou ${ }^{3}$, Shuiyuan Xiao ${ }^{1 *}$ and Junqun Fang ${ }^{4 *}$ \\ ${ }^{1}$ Department of Social Medicine and Health Management, Xiangya School of Public Health, Central South University, \\ Changsha, China, ${ }^{2}$ Department of Geriatric Medicine, Xiangya Hospital, Central South University, Changsha, China, \\ ${ }^{3}$ Research Center for Public Health and Social Security, School of Public Administration, Hunan University, Changsha, China, \\ ${ }^{4}$ Department of Health Management, Maternal and Child Health Hospital of Hunan Province, Changsha, China
}

Background: Antipsychotics contribute to the development of type 2 diabetes mellitus (T2DM) in individuals with schizophrenia. However, the extent of the relationship between antipsychotic use and T2DM varies in different settings, and the magnitude of the drug-specific effects fluctuates widely. This study aimed to explore the association of T2DM with antipsychotic use among enrollees with schizophrenia in China's National Basic Public Health Service Program (NBPHSP) and the drug-specific relationship with T2DM among patients receiving antipsychotic monotherapy.

Methods: We recruited diabetes-free patients with schizophrenia who were enrolled in the NBPHSP of Hunan Province from October 2009 to December 2018. The participants were classified into the following three groups: regular antipsychotic use, intermittent antipsychotic use, and antipsychotic-free groups. The patients were followed up until they received a T2DM diagnosis or until April 2019. Cox regression models were constructed to calculate the overall and drug-specific hazard ratios (HRs) to determine the antipsychotic-T2DM relationship. Interactive and subgroup analyses were performed to assess the heterogeneity of the effects across subgroups.

Results: A total of 122,064 NBPHSP enrollees with schizophrenia were followed up for 1,507,829 cumulative person-years, and 2,313 (1.89\%) patients developed T2DM. Patients who regularly and intermittently used antipsychotics had 117\% (HR: 2.17, 95\% Cl: 1.83-2.57) and 53\% (HR: 1.53, 95\% Cl: 1.23-1.90) higher risks of developing T2DM than antipsychotic-free patients, respectively. Regarding monotherapy, the T2DM risk increased by $66,80,62$, and $64 \%$ after the regular use of clozapine, risperidone, chlorpromazine, and perphenazine, respectively. In addition, the antipsychotic-related risk of T2DM decreased as the patient's baseline body mass index, and baseline fasting plasma glucose level, as well as the dietary proportion of animal products, increased.

Conclusion: Antipsychotics, especially clozapine, risperidone, chlorpromazine, and perphenazine, increased the T2DM risk among NBPHSP enrollees with schizophrenia. 
Mental health officers should accurately identify enrollees at a high risk of T2DM and take appropriate preventive measures to reduce the incidence of T2DM among patients with schizophrenia.

Keywords: schizophrenia, antipsychotic, type 2 diabetes mellitus, polytherapy, monotherapy

\section{INTRODUCTION}

Schizophrenia is a complex mental disorder that incurs a significant burden on patients, their families, and society at large. According to the Global Burden of Disease Study, there were an estimated 13.1 million patients with schizophrenia in 1990, and the number increased to 20.9 million in 2016 (1). Patients with schizophrenia have difficulty achieving both clinical and functional recovery (2), and their life expectancy is $\sim 20 \%$ lower than that of the general population (3). Type 2 diabetes mellitus (T2DM) is a common comorbidity in patients with schizophrenia that often results in an increased risk of early death $(4,5)$. The prevalence of T2DM is 2-3-fold higher among patients with schizophrenia than in the general population $(6,7)$. The reasons for this high prevalence are multifactorial and are of concern to both researchers and psychiatrists.

As genetic, environmental, and disease-specific factors contribute to the increased risk of $\mathrm{T} 2 \mathrm{DM}$ in patients with schizophrenia, the potential role of antipsychotics has been actively evaluated in T2DM onset. Although early evidence has been provided by pharmacoepidemiological studies (8-11), owing to biases and residual confounders, the links identified are weak and inconsistent $(11,12)$. Over the last decade, results from randomized clinical trials have also indicated the metabolic effects of antipsychotics; however, the duration of most trials was not long enough for diabetes to develop via its natural course $(8,13)$. Over the past 5 years, some well-designed clinical studies have confirmed the association between antipsychotic use and T2DM development (14). Nevertheless, the association between T2DM and antipsychotic use remains disputable for a number of reasons. First, psychopharmacological management of schizophrenia varies among patients of different racial, ethnic, or cultural backgrounds $(15,16)$. This results in differences in the duration of antipsychotic treatment and dosage, which may influence antipsychotic-related pro-diabetic effects $(10,17)$. Second, the risks of T2DM vary with different antipsychotic prescriptions as reported in different studies (13, 18, 19). Finally, different lifestyles and different severities of psychotic symptoms may also lead to inconsistent pro-diabetic effects of antipsychotics; this, however, still requires further verification. Thus, it is important to evaluate the association of T2DM with antipsychotic use in different real-world scenarios.

In China, the number of people with schizophrenia doubled between 1990 and 2010, from 3.09 to 7.16 million (20). In 2004, China heavily invested in the Central Government Support for the Local Management and Treatment of Severe Mental Illnesses Project (21), and individuals with schizophrenia accounted for $\sim 70 \%$ of the patients managed (22). In 2009, the project was incorporated into the National Basic Public Health Service Program (NBPHSP) (23), which encompasses 14 categories of public health services, including management of serious mental illness (SMI) and non-communicable diseases (NCDs). As of 2018, the program has managed and treated over four million patients with schizophrenia (22). Further elucidation of the association between antipsychotic use and T2DM among NBPHSP enrollees with schizophrenia in China can provide information for mental health officers to precisely evaluate the T2DM risk among antipsychotic users and accurately identify patients with schizophrenia at a high risk of T2DM. In addition, the results can provide recommendations for antipsychotic selection to mental health officers involved in the NBPHSP.

Therefore, this study was conducted to improve the identification of patients with schizophrenia at a high risk of T2DM in the NBPHSP by exploring (1) the relationship between antipsychotic use and T2DM among patients receiving monotherapy or polytherapy; (2) the drug-specific relationships between antipsychotics and T2DM among patients on monotherapy; and (3) the heterogeneity of the effects of antipsychotics on T2DM development among different subgroups of patients with schizophrenia.

\section{SUBJECTS AND METHODS}

\section{Study Population}

The present study was based on the SMI and NCDs management systems of the NBPHSP. The SMI management system serves all patients with SMI in a given jurisdiction. Once a patient with SMI is enrolled into the system, a medical team would collect patient information systematically, including sociodemographic characteristics, health-related behaviors, mental condition assessment, and health examination, which allows the establishment of a comprehensive health record. Once patients are enrolled in the system, they are visited by mental health officers every 3 months for risk assessment, mental condition examination, and medication adjustment, if needed. If a patient is out of contact, local administrative officials will help mental health officers to contact the patient and his/her caregivers. In this study, when a patient moved to another province, withdrew from the system, or was completely out of contact, he/she was regarded as lost to follow-up. For those under management, a comprehensive health examination is conducted every 6 months. When patients with elevated blood glucose are identified, they are referred to specialized hospitals for diagnosis and treatment. Those diagnosed with diabetes are registered in the NCDs management system to obtain further medical benefits from the government.

Participants in this study were patients who were diagnosed with schizophrenia and did not have diabetes at the time of diagnosis. Participants were enrolled in the SMI management system from October 2009 to December 2018 in Hunan Province. 
Patients with schizophrenia in the SMI management system were diagnosed by psychiatrists based on their medical history, clinical symptoms, and course of the disease according to ICD-10 (24). From 2009 to 2018, a total of 181,541 patients with schizophrenia were enrolled in the SMI system in Hunan Province. We excluded 55,374 patients whose records had logical errors, such as the date of the diagnosis earlier than the date of birth or the data of treatment earlier than that of the first episode. Furthermore, 1,556 patients who were diagnosed with diabetes prior to their schizophrenia diagnosis and 2,547 patients without medication information were excluded. Finally, 122,064 patients with schizophrenia were included and followed up in this study.

\section{Assessment of Antipsychotic Drug Use}

Antipsychotic use information was obtained from the SMI management system of the NBPHSP. Although several patients were diagnosed and began antipsychotic therapy before enrolling in the NBPHSP, mental health officers collected the information on the diagnosis, disease condition, and antipsychotic medication for all enrollees via medical records or face-to-face interviews with patients and their caregivers at the time of enrollment and during regular follow-up visits.

The participants were classified into the following three groups according to their use of antipsychotic drugs: regular antipsychotic use, intermittent antipsychotic use, and antipsychotic-free groups. Regular antipsychotic use referred to the continuous use of one or more antipsychotic drugs according to medical advice since the initial prescription; intermittent antipsychotic use referred to initial use of the antipsychotic $\operatorname{drug}(s)$, which was then discontinued because of patient's poor compliance; antipsychotic-free patients had not taken any antipsychotics since their diagnosis. The follow-up period was defined as the time from the first antipsychotic prescription for the regular and intermittent antipsychotic use groups and from the diagnosis of schizophrenia for the antipsychotic-free group to either the onset of T2DM or April 2019.

Patients who received monotherapy for the entire treatment course were also classified into regular antipsychotic use and intermittent antipsychotic use groups. Considering the sample size and statistical efficacy (25), we only included antipsychotic drugs that were used alone by more than 200 patients in each group, namely, clozapine, risperidone, chlorpromazine, perphenazine, olanzapine, sulpiride, and penfluridol.

\section{Assessment of T2DM}

The outcome of this study was the diagnosis of T2DM, which was obtained from the NCDs management (T2DM) system of the NBPHSP. T2DM was diagnosed by qualified physicians according to any of the following World Health Organization criteria $(26,27)$ : (1) typical symptoms of diabetes (polydipsia, polyuria, polyphagia, and weight loss) and a random blood glucose level $\geq 11.1 \mathrm{mmol} / \mathrm{l}(200 \mathrm{mg} / \mathrm{dl})$; (2) fasting plasma glucose $(\mathrm{FPG}) \geq 7.0 \mathrm{mmol} / \mathrm{l}(126 \mathrm{mg} / \mathrm{dl})$; (3) plasma glucose level $\geq 11.1 \mathrm{mmol} / \mathrm{l}(200 \mathrm{mg} / \mathrm{dl})$ in a 2 -h oral glucose tolerance test after a 75-g glucose load. Patients without diabetes symptoms were retested on a different day. Diabetes classification was carefully confirmed by physicians according to etiological characteristics and clinical features of the disease at the time of diagnosis.

\section{Assessment of Covariates}

Information on socio-demographic characteristics (age, sex, marital status, educational level, and occupation), health-related behaviors (smoking status, alcohol drinking frequency, diet pattern, and physical activity), and other potential confounding factors (insight and risk ratings) was collected by mental health officers using standardized questionnaires during faceto-face interviews with patients and their caregivers at the time of enrollment.

Three options each were offered to the responders regarding their marital status (unmarried, married, and divorced or widowed), educational level (primary school or below, middle school, and high school or above), and occupation (unemployed, agricultural worker, and other professions). Regarding healthrelated behaviors, responders were asked about the frequency of their physical activity (never, occasionally, once a week, or every day), alcohol drinking (seldom, occasionally, frequently, or every day), smoking status (non-smoker, current smoker, or former smoker), and diet pattern (plant-based diet, balanced plant- and animal-based diet, or animal-based diet). The insight was also classified into three categories as follows: complete insight meant that patients were aware of their mental disorder, thoroughly recognized the pathological manifestations, and understood the need for treatment; incomplete insight meant that patients were aware of their mental disorder but were unable to correctly recognize and analyze their pathological manifestations, and absent insight meant that patients were unaware of their mental disorder. Risk rating was categorized into three levels as follows: level 0 referred to patients who exhibited no dangerous behavior; level 1 referred to patients who made verbal threats and/or shouted but did not act physically; level 2 or higher referred to patients who exhibited physically abusive behaviors.

At the time of enrollment, the height and weight were measured using a calibrated height and weight gauge. The body mass index $\left(\mathrm{BMI}, \mathrm{kg} / \mathrm{m}^{2}\right)$ was calculated by dividing weight $(\mathrm{kg})$ by squared height $(\mathrm{m})$. FPG, serum albumin, serum creatinine, and serum nitrogen were tested using standard clinical chemistry methods.

\section{Data Quality Management}

The process of data collection and database management was supervised by full-time staff, and spot checks were conducted according to the work manual. Data were extracted from the database by a statistician under close supervision to ensure data quality. All data sheets were checked and evaluated for completeness after collection.

\section{Statistical Analysis}

The baseline characteristics of the participants were presented as the mean \pm standard deviation for continuous variables and as $n$ (\%) for categorical variables. ANOVA was performed for continuous variables, and a chi-square test was performed for categorical variables to compare differences in baseline 


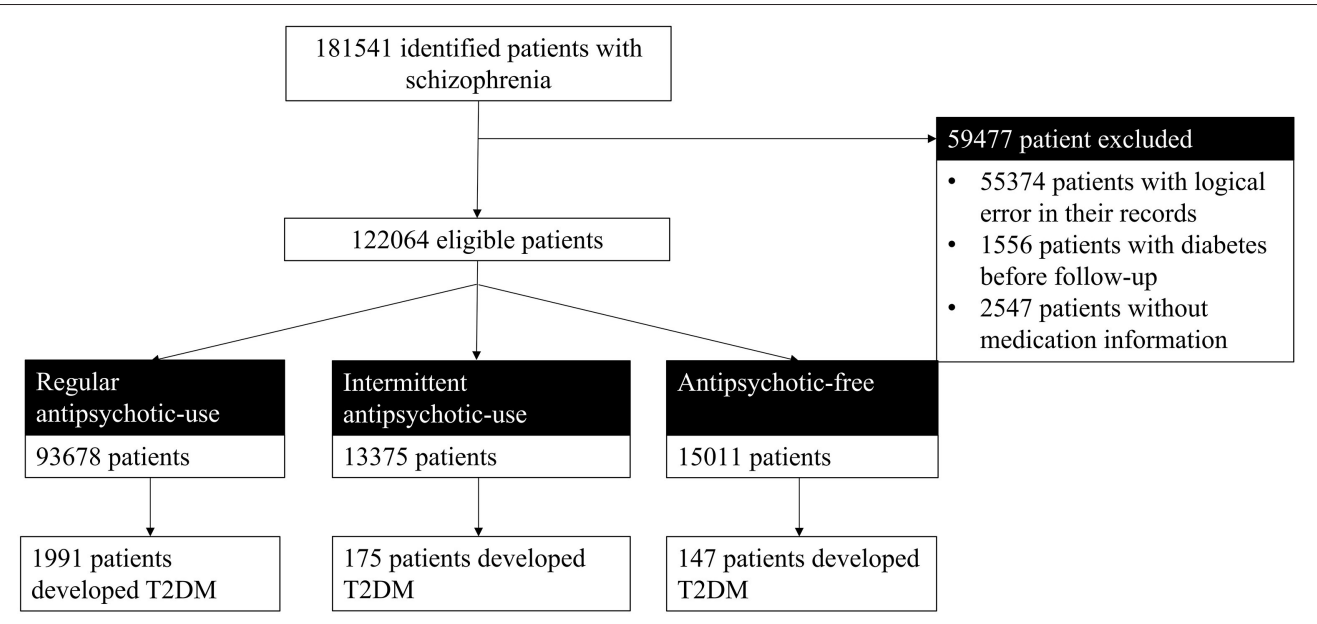

FIGURE 1 | Flowchart of the inclusion of participants. T2DM, type 2 diabetes mellitus.

characteristics among the groups. Kaplan-Meier survival curves were plotted to determine the timeline of T2DM development in accordance with the use of antipsychotic drugs. The log-rank test was used to compare the T2DM-free rates in the different groups. Three Cox proportional hazard regression models were constructed to calculate the crude and adjusted hazard ratios (HRs) to determine the association between antipsychotic use and T2DM (Model 1: adjusting for age and sex; model 2: adjusting for age, sex, baseline BMI, and baseline FPG; and model 3: adjusting for age, sex, baseline BMI, baseline FPG, family history of diabetes, educational level, occupation, marital status, smoking status, alcohol drinking frequency, diet pattern, physical activity, insight rating, and risk rating). The outcome in the three models was the incidence of T2DM. The time variable was defined as the duration from the first antipsychotic prescription for the regular and intermittent antipsychotic use groups and from the diagnosis of schizophrenia for the antipsychotic-free group to either the onset of T2DM or April 2019. Furthermore, the drug-specific T2DM HRs of different antipsychotics were calculated after adjusting for all variables included in model 3. Interactive and subgroup analyses were conducted to further assess the heterogeneity of the effects across the subgroups. For the sensitivity analysis, we included baseline albumin, creatinine, and nitrogen levels in the Cox regression model to verify the robustness of the results.

Data were analyzed using the SAS statistical package (version 9.4 SAS Institute, Inc., Cary, North Carolina, USA), and graphs were plotted in $\mathrm{R}$ (version 4.1.0). All tests were two-sided, and statistical significance was set at $P<0.05$. In the subgroup analysis, the significant levels were adjusted by the Bonferroni method to minimize the type I error.

\section{RESULTS}

\section{Population Characteristics}

A total of 122,064 patients with schizophrenia who were under the management of the NBPHSP were followed up for
$1,507,829$ cumulative person-years. The median duration of the follow-up was 9.16 years (interquartile range: 4.75-18.04 years). Regarding antipsychotic use, 93,678 patients (88.04\%) used antipsychotics continuously; 13,375 patients $(10.96 \%)$ used antipsychotics intermittently, and 15,011 patients $(12.30 \%)$ were antipsychotic-free (Figure 1).

A summary of the baseline characteristics of the patients is presented in Table 1. The average ages of patients with regular antipsychotic use, those with intermittent antipsychotic use, and antipsychotic-free patients were $33.26 \pm 13.26,35.26 \pm 12.61$, and $45.48 \pm 12.30$ years, respectively; the percentages of male patients in the three groups were $49.82 \%, 48.08 \%$, and $50.5 \%$, respectively; the average baseline BMIs in the three groups were $22.52 \pm 2.99,22.21 \pm 2.89$, and $22.14 \pm 2.82 \mathrm{~kg} / \mathrm{m}^{2}$, respectively; and the average baseline FPG levels were 5.14 $\pm 0.61,5.12 \pm 0.60$, and $5.13 \pm 0.60 \mathrm{mmol} / \mathrm{L}$, respectively. Significant differences were found in all characteristics among the three groups (Table 1).

\section{Effects of Antipsychotics on T2DM Development}

In total, 2,313 (1.89\%) patients developed diabetes in this cohort. In the regular and intermittent antipsychotic use groups, 1,991 $(2.12 \%)$ and $175(1.32 \%)$ patients with schizophrenia developed T2DM, respectively. In the antipsychotic-free group, only 147 (0.99\%) patients with schizophrenia developed T2DM.

The Kaplan-Meier curves demonstrated the probabilities of being T2DM-free in different groups (Figure 2). There was a significant difference in the probabilities, with a lower probability of being T2DM free associated with an increased frequency of antipsychotics use (log-rank test, $P<0.001$ ).

\section{Hazard Ratio for the Development of T2DM}

In model 1, compared with those in the antipsychotic-free group, individuals who continuously used antipsychotics had a $167 \%$ higher risk of developing T2DM (HR: 2.67, 95\% CI: 2.263.16), and individuals who intermittently used antipsychotics 
TABLE 1 | Characteristics of participants with different antipsychotic use conditions.

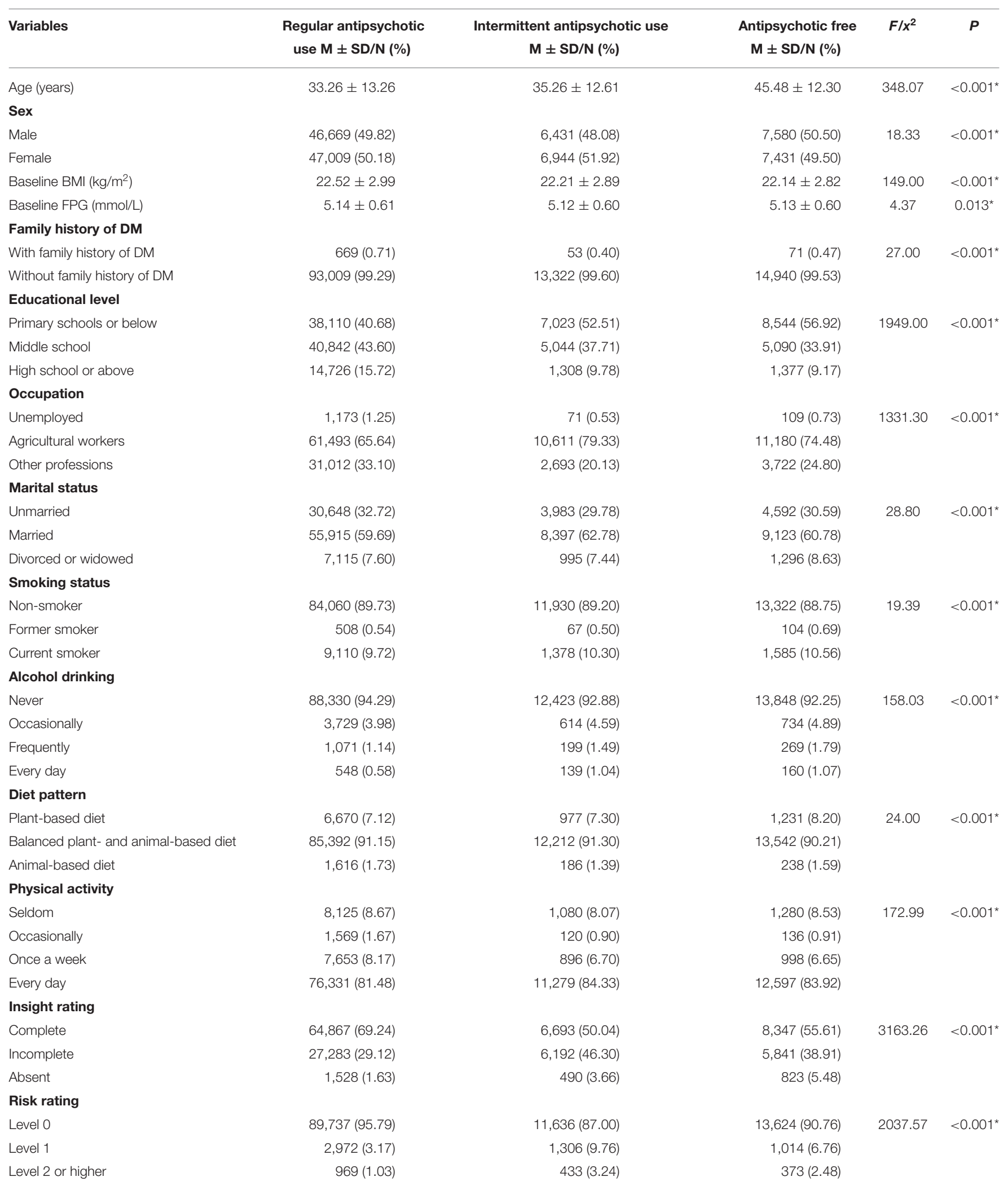

${ }^{\star} P$ < 0.05. M, mean; SD, standard deviation; BMI, body mass index; FPG, fasting plasma glucose; kg, kilogram; $m$, meter; DM, diabetes mellitus. 


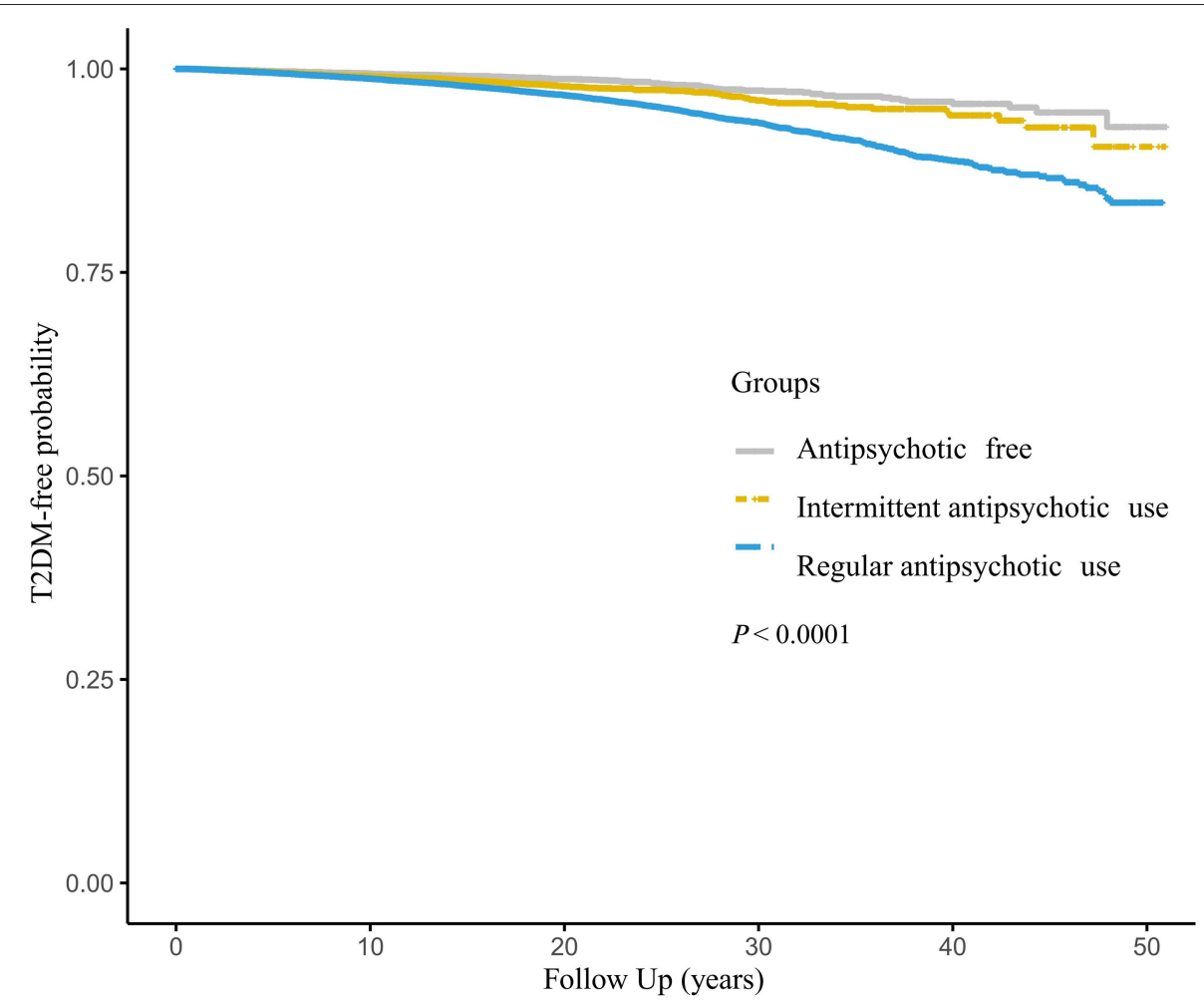

FIGURE 2 | Kaplan-Meier analysis of T2DM by antipsychotic use. T2DM, type 2 diabetes mellitus.

had a 52\% higher risk of developing T2DM (HR: 1.52, 95\% CI: $1.22-1.90)$. In model 2 , individuals who continuously used antipsychotics and those who intermittently used antipsychotics had a $141 \%$ (HR: 2.41, 95\% CI: 2.04-2.85) and 51\% (HR: 1.51, 95\% CI: 1.21-1.88) higher risk of developing T2DM than those in the antipsychotic-free group, respectively. In model 3, the risk of developing T2DM was $117 \%$ higher in the patients who continuously used antipsychotics (HR: 2.17, 95\% CI: 1.83-2.57) and $53 \%$ higher in the individuals who intermittently used antipsychotics than in those from the antipsychotic-free group (HR: 1.53, 95\% CI: 1.23-1.90) (Table 2).

With regard to monotherapy, the risk for developing T2DM increased following the regular use of clozapine by $66 \%$ (HR: 1.66, 95\% CI: $1.35-2.04$ ), risperidone by $80 \%$ (HR: $1.80,95 \%$ CI: $1.41-2.30$ ), chlorpromazine by $62 \%$ (HR: $1.62,95 \%$ CI: $1.21-$ 2.16 ), and perphenazine by $64 \%$ (HR: $1.64,95 \%$ CI: 1.15-2.33). The T2DM hazard ratios were not statistically significant after the regular use of olanzapine (HR: 1.09, 95\% CI: 0.68-1.73), sulpiride (HR: 1.14, 95\% CI: 0.73-1.78), and penfluridol (HR: 1.40, 95\% CI: 0.83-2.35) (Figure 3).

\section{Interactive, Subgroup, and Sensitivity Analyses}

In the interactive analysis, the effects of interactions between antipsychotic use and the baseline BMI, baseline FPG level, and diet pattern on the development of T2DM were statistically significant $(P<0.05)$. None of the interactions between other factors (age, sex, alcohol drinking, smoking status, physical activity, insight rating, risk rating, and family history of diabetes) and antipsychotic use on the development of T2DM were statistically significant. In the subgroup analysis, the risk of T2DM with antipsychotic use decreased as the baseline BMI and baseline FPG level, as well as the proportion of animal products in the patient's diet, increased (Supplementary Table 1). Similar trends were observed for patients treated with monotherapy (Supplementary Figure 1). In the sensitivity analysis, the results were consistent with those of models 1-3 (Supplementary Table 2).

\section{DISCUSSION}

\section{Main Findings}

In the present study, we found that regular antipsychotic use was associated with a $117 \%$ higher risk of developing T2DM among NBPHSP enrollees with schizophrenia. Regarding monotherapy, the T2DM risk increased by $66 \%, 80 \%, 62 \%$ and $64 \%$ after the regular use of clozapine, risperidone, chlorpromazine, and perphenazine, respectively. The risk of developing T2DM due to antipsychotic use decreased with an increase in the patient's baseline BMI and baseline FPG level, as well as the proportion of animal products in the diet. The strengths of this study include a large sample size and a long follow-up period based on the NBPHSP data in China, as well as a comprehensive exploration of the heterogeneity of the effects of antipsychotics on T2DM 
TABLE 2 | Hazard ratios of T2DM according to antipsychotic use.

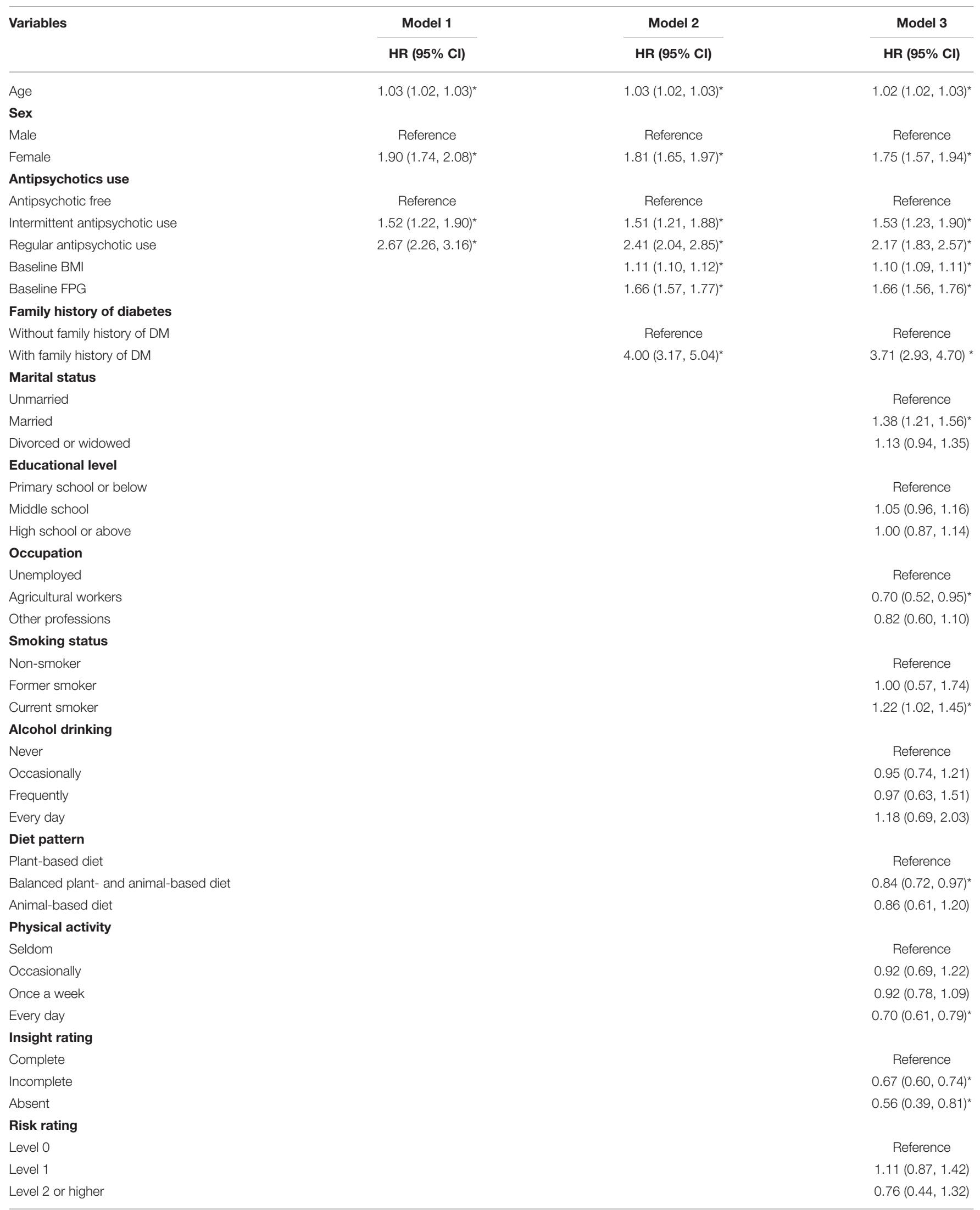

${ }^{*} P<0.05$. DM, diabetes mellitus; T2DM, type 2 diabetes mellitus; HR, hazard ratio; Cl, confidence interval; BMI, body mass index; FPG, fasting plasma glucose. 


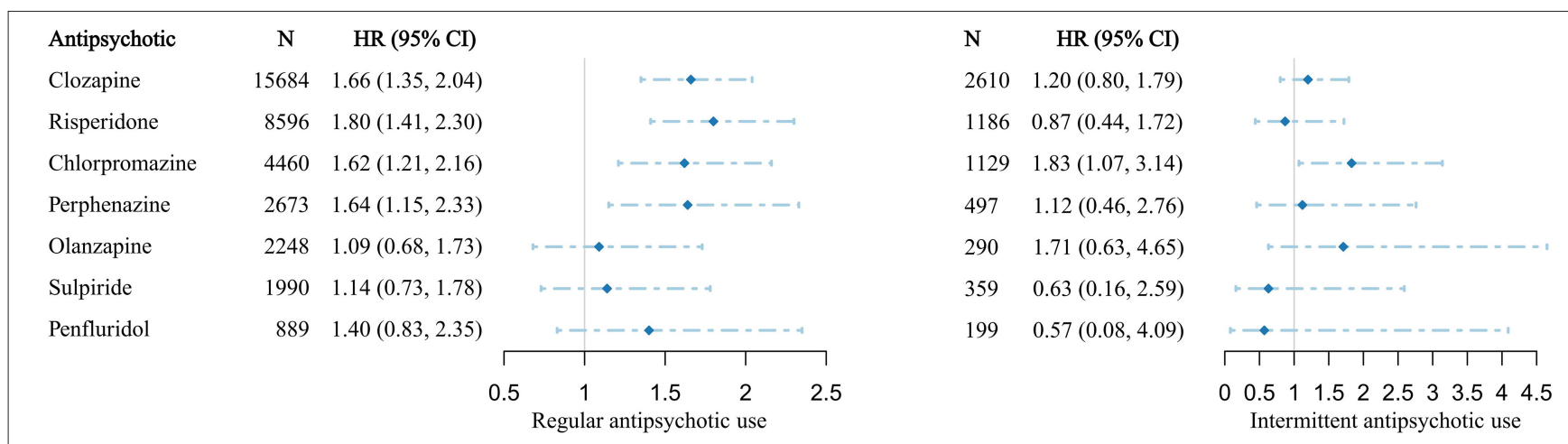

FIGURE 3 | Antipsychotic-related hazard ratios of T2DM in patients with schizophrenia receiving monotherapy. HR, hazard ratio; Cl, confidence interval. HRs and 95\% Cls were calculated by multivariate-adjusted Cox regression model.

development among different subgroups of patients. This study provides information for identifying patients at a high risk of T2DM in the SMI system of the NBPHSP and for developing strategies to prevent the development of T2DM among patients with antipsychotic prescriptions.

\section{Comparison With Similar Studies and Interpretations}

In this study, we found that patients with schizophrenia who regularly or intermittently took antipsychotics had a significantly higher risk of developing T2DM than antipsychotic-free patients, which was consistent with the results of previous studies $(7,19$, 28-30). The T2DM risk was higher in antipsychotic users than in antipsychotic-free controls $(10,19,30)$, for which there are several potential reasons. For instance, the association between antipsychotics and diabetes is believed to be mediated by weight gain $(31,32)$. The increased risk of diabetes may also be associated with insulin resistance, reduced insulin sensitivity, and abnormal blood glucose regulation, which is thought to be mediated by muscarinic M3 receptor antagonism (33).

Regarding monotherapy, patients who regularly used clozapine, risperidone, chlorpromazine, and perphenazine had a significantly higher risk of developing T2DM than antipsychoticfree patients. According to previous studies, the pro-diabetic effects differ for different antipsychotics, and the extent of this risk varies widely $(13,19)$. Some studies have reported a higher prevalence of T2DM among users of all categories of antipsychotic medications than among antipsychotic-free participants $(7,28)$. In particular, clozapine and olanzapine were identified as the top two diabetes-causing agents $(34,35)$. However, the relationship of diabetes with olanzapine use was found to be insignificant in two population-based studies $(10,19)$. The olanzapine-caused increase in blood sugar levels can be reversed by discontinuing olanzapine (36). Since olanzapine demonstrated a stronger obesogenic effect than that of most other antipsychotics $(31,37)$, we cannot rule out the possibility that some olanzapine users switched to other antipsychotics or stopped using antipsychotic drugs entirely because of the significant weight gain (38), which may have resulted in the insignificant relationship between olanzapine and T2DM in this study.
In this study, the risk of antipsychotic-related T2DM decreased as the patient's baseline BMI and baseline FPG increased, which may be attributed to the efforts of mental health staff in the NBPHSP. As reported in previous studies, patients with a high baseline BMI and FPG level were at a higher risk of diabetes $(39,40)$. Regarding those with a high risk of developing T2DM, according to the recommendation in the Chinese Schizophrenia Prevention and Control Guideline (41) and the requirement in the National Basic Public Health Service Specifications (42), psychiatrists should initiate a lifestyle intervention at the beginning of treatment, helping patients adjust their diets and maintain physical activity. In addition, psychiatrists were requested to try to choose antipsychotics with fewer metabolic side effects and prescribe medications that help prevent diabetes, such as metformin or orlistat, when necessary (43). Furthermore, an increase in BMI, regardless of the baseline BMI, is an independent risk factor of diabetes (44). In the case of a relatively high BMI and FPG level, psychiatrists, caregivers, and patients would make efforts to keep the BMI and FPG stable, which could buffer the pro-diabetic effects of antipsychotics, to some extent.

The association between antipsychotics and T2DM in patients on balanced plant- and animal-based or animal-based diets was lower than that in patients on a plant-based diet. People with schizophrenia often have a low socioeconomic status and income (45), which makes it difficult for them to obtain highquality food. Although plant-based diets have been proven to be protective against diabetes, low-quality plant-based diets may lead to protein deficiency in patients with schizophrenia, making it difficult for the body to maintain homeostasis in response to the metabolic side effects of antipsychotics (46). A high-quality diet with an adequate amount of protein is effective in improving blood glucose control (39). As recommended by the Canadian Diabetes Association in 2013, both the quantity and quality of protein intake must be optimized to meet the requirements for essential amino acids to prevent the onset of T2DM (47). In addition, patients whose diets include a high proportion of animal products consume more heme iron (48). Iron constitutes the metal nucleus of many cellular enzymes and is important for most cellular processes, including $\beta$-cell metabolism and insulin secretion (49), which help in glycemic regulation. Hence, 
a proper diet with sufficient animal products and adequate highquality plant foods might help mitigate the pro-diabetic side effects of antipsychotics.

\section{Implications of the Findings for the Prevention and Treatment of T2DM}

There are several practical implications of our findings. First, mental health officers involved in the NBPHSP should provide dietary and behavioral guidance, along with antipsychotic prescriptions, to patients with schizophrenia to help reduce T2DM risks (50). Second, mental health officers should conduct a regular blood glucose screening of patients with schizophrenia, especially those who regularly use antipsychotics. With a timely risk assessment and comprehensive intervention strategy, mental health officers and caregivers can help patients with schizophrenia reduce the risk of T2DM, which also reduces the risks of secondary cardiovascular disease and the associated mortality (50). Third, ensuring adequate amounts of highquality animal products in the diet can help patients maintain glucometabolic homeostasis in response to antipsychotics. Lastly, the existing preventive measures for patients at a high risk of diabetes are effective to some extent. However, further implementation of these measures is needed, along with more attention to groups with a lower risk of diabetes.

\section{Limitations and Future Research}

There are several limitations to this study. First, the assessment of antipsychotic drug use was only based on medical records and face-to-face interviews, but it was not confirmed by serum level measurements. To avoid potential information bias, we should consider serum level measurements of antipsychotic medications in future research. Second, the dosage of an antipsychotic drug affects the risk of developing T2DM (51); however, detailed information on the dosages was not collected in this program, which made our results only a rough estimate. Third, information related to T2DM diagnosis was obtained from the NCDs management system of the NBPHSP. Since the proportion of antipsychotic users with undiagnosed diabetes was considerable (52), this study inevitably underestimated the incidence of T2DM. In addition, patients with schizophrenia who do not require medication may have less connection with the system. This may lead to under-diagnosis of T2DM in the antipsychoticfree group and, therefore, exaggerate the effects of antipsychotics on the development of T2DM. Fourth, we were unable to control some potential confounders, such as a sedentary lifestyle, because of the lack of information. Thus, residual confounding factors may have influenced the study findings. Lastly, the antipsychoticinduced weight gain or blood glucose increase might lead patients to discontinue therapy, which would cause an inevitable survival bias (38). Despite these limitations, our findings revealed the actual risks of antipsychotics-induced T2DM in real-world practice.

It is crucial to conduct further research to prevent T2DM among antipsychotic users. The contribution of various known risk factors, such as a BMI change and diet pattern, to the association of T2DM with antipsychotic use warrants further exploration. In addition, antipsychotics with fewer metabolic side effects are yet to be developed. In the selection of the treatment strategy, efficacy should be the most important consideration. Further studies should focus on exploring optimal treatment strategies that balance both therapeutic efficacy and metabolic risk management not only in the NBPHSP but also in different scenarios.

\section{CONCLUSIONS}

Antipsychotics, especially clozapine, risperidone, chlorpromazine, and perphenazine, increased the risk of T2DM in enrollees with schizophrenia in the NBPHSP. However, the risk decreased as the patient's baseline BMI and baseline FPG level, as well as the proportion of animal products in the patient's diet, increased. For patients at a high risk of developing T2DM, mental health workers should provide regular assessments of glucose levels, recommend lifestyle modifications, and adjust medication prescriptions when necessary.

\section{DATA AVAILABILITY STATEMENT}

The generated datasets are available after the approval of National Basic Public Health Service Program of Hunan Province, China. Introduction to the program and detailed information of the application can be found in http://www.nbphsp.org.cn/. Further inquiries can be directed to the corresponding author/s.

\section{ETHICS STATEMENT}

This study involving human participants was reviewed and approved by the institutional review board of Xiangya School of Public Health, Central South University (NO. XYGW-2021-41).

\section{AUTHOR CONTRIBUTIONS}

FO, SX, and JF contributed to the conception and design of the study. FO and $\mathrm{JH}$ performed the statistical analysis and wrote the first draft of the manuscript. WZ, XC, and SX reviewed and edited the manuscript. All authors read and approved the final manuscript.

\section{FUNDING}

This work was supported by the National Key Research and Development Program of China (Grant No: 2016YFC0900802).

\section{ACKNOWLEDGMENTS}

We acknowledge Caixia Feng for her important participation and contribution.

\section{SUPPLEMENTARY MATERIAL}

The Supplementary Material for this article can be found online at: https://www.frontiersin.org/articles/10.3389/fpsyt. 2022.754775/full\#supplementary-material 


\section{REFERENCES}

1. Charlson FJ, Ferrari AJ, Santomauro DF, Diminic S, Stockings E, Scott JG, et al. Global epidemiology and burden of Schizophrenia: findings from the global burden of disease study 2016. Schizophr Bull. (2018) 44:1195203. doi: $10.1093 /$ schbul/sby 058

2. Jääskeläinen E, Juola P, Hirvonen N, McGrath JJ, Saha S, Isohanni M, et al. A systematic review and meta-analysis of recovery in Schizophrenia. Schizophr Bull. (2013) 39:1296-306. doi: 10.1093/schbul/sbs130

3. Saha S, Chant D, McGrath J. A systematic review of mortality in Schizophrenia: is the differential mortality gap worsening over time? Arch Gen Psychiatry. (2007) 64:1123-31. doi: 10.1001/archpsyc.64.10.1123

4. Hennekens CH, Hennekens AR, Hollar D, Casey DE. Schizophrenia and increased risks of cardiovascular disease. Am Heart J. (2005) 150:111521. doi: 10.1016/j.ahj.2005.02.007

5. Suvisaari J, Partti K, Perälä J, Viertiö S, Saarni SE, Lönnqvist J, et al. Mortality and its determinants in people with psychotic disorder. Psychosom Med. (2013) 75:60-7. doi: 10.1097/PSY.0b013e31827ad512

6. Vancampfort D, Wampers M, Mitchell AJ, Correll CU, De Herdt A, Probst $M$, et al. A meta-analysis of cardio-metabolic abnormalities in drug naïve, first-episode and multi-episode patients with Schizophrenia versus general population controls. World Psychiatry. (2013) 12:24050. doi: 10.1002/wps.20069

7. Rajkumar AP, Horsdal HT, Wimberley T, Cohen D, Mors O, Børglum AD, et al. Endogenous and antipsychotic-related risks for diabetes mellitus in young people with Schizophrenia: a Danish population-based cohort study. Am J Psychiatry. (2017) 174:686-94. doi: 10.1176/appi.ajp.2016.16040442

8. McEvoy JP, Meyer JM, Goff DC, Nasrallah HA, Davis SM, Sullivan $\mathrm{L}$, et al. Prevalence of the metabolic syndrome in patients with schizophrenia: baseline results from the Clinical Antipsychotic Trials of Intervention Effectiveness (CATIE) Schizophrenia trial and comparison with national estimates from NHANES III. Schizophr Res. (2005) 80:19-32. doi: 10.1016/j.schres.2005.07.014

9. Polcwiartek C, Kragholm K, Rohde C, Hashemi N, Vang T, Nielsen J. Diabetic ketoacidosis and diabetes associated with antipsychotic exposure among a previously diabetes-naive population with schizophrenia: a nationwide nested case-control study. Diabetologia. (2017) 60:1678-90. doi: 10.1007/s00125-017-4320-5

10. Nishtala PS, Chyou TY. Real-world risk of diabetes with antipsychotic use in older New Zealanders: a case-crossover study. Eur J Clin Pharmacol. (2017) 73:233-9. doi: 10.1007/s00228-016-2158-2

11. Rondanelli M, Sarra S, Antoniello N, Mansi V, Govoni S, Falvo F, et al. No effect of atypical antipsychotic drugs on weight gain and risk of developing type II diabetes or lipid abnormalities among nursing home elderly patients with Alzheimer's disease. Minerva Med. (2006) 97:147-51.

12. Holt RI, Peveler RC. Antipsychotic drugs and diabetes-an application of the Austin Bradford Hill criteria. Diabetologia. (2006) 49:146776. doi: 10.1007/s00125-006-0279-3

13. Holt RIG. Association between antipsychotic medication use and diabetes. Curr Diab Rep. (2019) 19:96. doi: 10.1007/s11892-019-1220-8

14. Ingimarsson $\mathrm{O}, \mathrm{MacCabe} \mathrm{JH}$, Haraldsson M, Jónsdóttir H, Sigurdsson E. Risk of diabetes and dyslipidemia during clozapine and other antipsychotic drug treatment of schizophrenia in Iceland. Nord J Psychiatry. (2017) 71:496502. doi: 10.1080/08039488.2017.1334821

15. Kreyenbuhl J, Zito JM, Buchanan RW, Soeken KL, Lehman AF. Racial disparity in the pharmacological management of Schizophrenia. Schizophr Bull. (2003) 29:183-93. doi: 10.1093/oxfordjournals.schbul.a006996

16. Puyat JH, Daw JR, Cunningham CM, Law MR, Wong ST, Greyson DL, et al. Racial and ethnic disparities in the use of antipsychotic medication: a systematic review and meta-analysis. Soc Psychiatry Psychiatr Epidemiol. (2013) 48:1861-72. doi: 10.1007/s00127-013-0753-4

17. Mangurian C, Keenan W, Newcomer JW, Vittinghoff E, Creasman JM, Schillinger D. Diabetes prevalence among racial-ethnic minority group members with severe mental illness taking antipsychotics: double jeopardy? Psychiatr Serv. (2017) 68:843-6. doi: 10.1176/appi.ps.201600356

18. Hirsch L, Yang J, Bresee L, Jette N, Patten S, Pringsheim T. Second-generation antipsychotics and metabolic side effects: a systematic review of populationbased studies. Drug Saf. (2017) 40:771-81. doi: 10.1007/s40264-017-0543-0
19. Foley DL, Mackinnon A, Morgan VA, Watts GF, Castle DJ, Waterreus A, et al. Effect of age, family history of diabetes, and antipsychotic drug treatment on risk of diabetes in people with psychosis: a population-based cross-sectional study. Lancet Psychiatry. (2015) 2:1092-8. doi: 10.1016/S2215-0366(15)00276-X

20. Chan KY, Zhao FF, Meng S, Demaio AR, Reed C, Theodoratou E, et al. Prevalence of Schizophrenia in China between 1990 and 2010. J Glob Health. (2015) 5:010410. doi: 10.1002/wps.20222

21. Ma H. Integration of hospital and community services-the '686 Project'-is a crucial component in the reform of China's mental health services. Shanghai Arch Psychiatry. (2012) 24:172-4. doi: 10.3969/j.issn.1002-0829.2012.03.007

22. Wang X, Ma N, Wu X, Zhang W, Guan L, Ma H, et al. Management and services for psychosis in People's Republic of China in 2018. Chin J Psychiatry. (2020) 53:438-45. doi: 10.3760/cma.j.cn113661-20200622-00290

23. Wang L, Wang Z, Ma Q, Fang G, Yang J. The development and reform of public health in China from 1949 to 2019. Global Health. (2019) 15:45. doi: 10.1186/s12992-019-0486-6

24. World Health Organization. The ICD-10 Classification of Mental and Behavioural Disorders-Diagnostic Criteria for Research. Geneva, Switzerland: World Health Organization (1992).

25. Lachin JM. Sample size and power for a logrank test and Cox proportional hazards model with multiple groups and strata, or a quantitative covariate with multiple strata. Stat Med. (2013) 32:4413-25. doi: 10.1002/sim.5839

26. World Health Organization. Definition, Diagnosis and Classification of Diabetes Mellitus and Its Complications: Report of a WHO Consultation. Part 1, Diagnosis and Classification of Diabetes Mellitus. Geneva: World Health Organization (1999).

27. Weng J, Ji L, Jia W, Lu J, Zhou Z, Zou D, et al. Standards of care for type 2 diabetes in China. Diabetes Metab Res Rev. (2016) 32:44258. doi: $10.1002 / \mathrm{dmr} .2827$

28. Suvisaari J, Perälä J, Saarni SI, Härkänen T, Pirkola S, Joukamaa M, et al. Type 2 diabetes among persons with schizophrenia and other psychotic disorders in a general population survey. Eur Arch Psychiatry Clin Neurosci. (2008) 258:129-36. doi: 10.1007/s00406-007-0762-y

29. Vancampfort D, Correll CU, Galling B, Probst M, De Hert M, Ward PB, et al. Diabetes mellitus in people with schizophrenia, bipolar disorder and major depressive disorder: a systematic review and large scale meta-analysis. World Psychiatry. (2016) 15:166-74. doi: 10.1002/wps.20309

30. Galling B, Roldán A, Nielsen RE, Nielsen J, Gerhard T, Carbon M, et al. Type 2 diabetes mellitus in youth exposed to antipsychotics: a systematic review and meta-analysis. JAMA Psychiatry. (2016) 73:24759. doi: 10.1001/jamapsychiatry.2015.2923

31. Leucht S, Cipriani A, Spineli L, Mavridis D, Orey D, Richter F, et al. Comparative efficacy and tolerability of 15 antipsychotic drugs in schizophrenia: a multiple-treatments meta-analysis. Lancet. (2013) 382:95162. doi: 10.1016/S0140-6736(13)60733-3

32. Correll CU, Detraux J, De Lepeleire J, De Hert M. Effects of antipsychotics, antidepressants and mood stabilizers on risk for physical diseases in people with schizophrenia, depression and bipolar disorder. World Psychiatry. (2015) 14:119-36. doi: $10.1002 /$ wps.20204

33. Deng C. Effects of antipsychotic medications on appetite, weight, and insulin resistance. Endocrinol Metab Clin North Am. (2013) 42:54563. doi: 10.1016/j.ecl.2013.05.006

34. Rummel-Kluge C, Komossa K, Schwarz S, Hunger H, Schmid F, Lobos CA, et al. Head-to-head comparisons of metabolic side effects of second generation antipsychotics in the treatment of schizophrenia: a systematic review and meta-analysis. Schizophr Res. (2010) 123:22533. doi: 10.1016/j.schres.2010.07.012

35. Kessing LV, Thomsen AF, Mogensen UB, Andersen PK. Treatment with antipsychotics and the risk of diabetes in clinical practice. Br J Psychiatry. (2010) 197:266-71. doi: 10.1192/bjp.bp.109.076935

36. Folnegović-Smalc V, Jukić V, Kozumplik O, Mimica N, Uzun S. Olanzapine use in a patient with schizophrenia and the risk of diabetes. Eur Psychiatry. (2004) 19:62-4. doi: 10.1016/j.eurpsy.2003.09.003

37. Fleischhaker C, Heiser P, Hennighausen K, Herpertz-Dahlmann B, Holtkamp $\mathrm{K}$, Mehler-Wex C, et al. Weight gain in children and adolescents during 45 weeks treatment with clozapine, olanzapine and risperidone. J Neural Transm. (2008) 115:1599-608. doi: 10.1007/s00702-008-0105-9 
38. Rico-Villademoros F, Calandre EP. Risk of bias in observational studies of interventions: the case of antipsychotic-induced diabetes. Lancet Psychiatry. (2016) 3:103-4. doi: 10.1016/S2215-0366(15)00541-6

39. Ley SH, Hamdy O, Mohan V, Hu FB. Prevention and management of type 2 diabetes: dietary components and nutritional strategies. Lancet. (2014) 383:1999-2007. doi: 10.1016/S0140-6736(14)60613-9

40. Preiss D, Welsh P, Murray HM, Shepherd J, Packard C, Macfarlane P, et al. Fasting plasma glucose in non-diabetic participants and the risk for incident cardiovascular events, diabetes, and mortality: results from WOSCOPS 15year follow-up. Eur Heart J. (2010) 31:1230-6. doi: 10.1093/eurheartj/ehq095

41. Shu L. Chinese Schizophrenia Prevention and Control Guideline. Beijing: Peking University Medical Press (2007).

42. The National Health Commission of the People's Republic of China. National Basic Public Health Service Specifications, 3rd Edn. Beijin (2017).

43. Holt RI. The prevention of diabetes and cardiovascular disease in people with Schizophrenia. Acta Psychiatr Scand. (2015) 132:86-96. doi: 10.1111/acps.12443

44. Suvisaari J, Keinänen J, Eskelinen S, Mantere O. Diabetes and Schizophrenia. Curr Diab Rep. (2016) 16:16. doi: 10.1007/s11892-015-0704-4

45. Perälä J, Saarni SI, Ostamo A, Pirkola S, Haukka J, Härkänen T, et al. Geographic variation and sociodemographic characteristics of psychotic disorders in Finland. Schizophr Res. (2008) 106:33747. doi: 10.1016/j.schres.2008.08.017

46. Evert AB, Boucher JL, Cypress M, Dunbar SA, Franz MJ, Mayer-Davis EJ, et al. Nutrition therapy recommendations for the management of adults with diabetes. Diabetes Care. (2014) 37:S120-43. doi: 10.2337/dc14-S120

47. Dworatzek PD, Arcudi K, Gougeon R, Husein N, Sievenpiper JL, Williams SL. Nutrition therapy. Can J Diabetes. (2013) 37:S45-55. doi: 10.1016/j.jcjd.2013.01.019

48. He J, Fang A, Yu S, Shen X, Li K. Dietary nonheme, heme, and total iron intake and the risk of diabetes in adults: results from the China health and nutrition survey. Diabetes Care. (2020) 43:776-84. doi: 10.2337/dc19-2202

49. Hansen JB, Moen IW, Mandrup-Poulsen T. Iron: the hard player in diabetes pathophysiology. Acta Physiol. (2014) 210:717-32. doi: 10.1111/apha.12256
50. American Diabetes Association, American Psychiatric Association, American Association of Clinical Endocrinologists, North American Association for the Study of Obesity. Consensus development conference on antipsychotic drugs and obesity and diabetes. Diabetes Care. (2004) 27:596-601. doi: 10.2337/diacare.27. 2.596

51. Ulcickas Yood M, Delorenze GN, Quesenberry CP Jr, Oliveria SA, Tsai AL, et al. Association between second-generation antipsychotics and newly diagnosed treated diabetes mellitus: does the effect differ by dose? BMC Psychiatry. (2011) 11:197. doi: 10.1186/1471-24 4X-11-197

52. Hirigo AT, Teshome T. The magnitude of undiagnosed diabetes and Hypertension among adult psychiatric patients receiving antipsychotic treatment. Diabetol Metab Syndr. (2020) 12:79. doi: 10.1186/s13098-020-00588-2

Conflict of Interest: The authors declare that the research was conducted in the absence of any commercial or financial relationships that could be construed as a potential conflict of interest.

Publisher's Note: All claims expressed in this article are solely those of the authors and do not necessarily represent those of their affiliated organizations, or those of the publisher, the editors and the reviewers. Any product that may be evaluated in this article, or claim that may be made by its manufacturer, is not guaranteed or endorsed by the publisher.

Copyright ( 2022 Ouyang, He, Cheng, Zhou, Xiao and Fang. This is an open-access article distributed under the terms of the Creative Commons Attribution License (CC $B Y)$. The use, distribution or reproduction in other forums is permitted, provided the original author(s) and the copyright owner(s) are credited and that the original publication in this journal is cited, in accordance with accepted academic practice. No use, distribution or reproduction is permitted which does not comply with these terms. 